\title{
A Database of Glyphs for OCR of Mathematical Documents
}

\author{
Alan Sexton and Volker Sorge \\ School of Computer Science, University of Birmingham, UK, \\ A.P.Sexton|V.Sorge@cs.bham.ac.uk, http://www.cs.bham.ac.uk/〜aps| vxs
}

\begin{abstract}
Automatic document analysis tools for mathematical texts are necessary to enlarge the pool of mathematical knowledge available in electronic form. However, development of such tools is currently hindered by the weakness of optical character recognition systems in dealing with the large range of mathematical symbols and the often subtle but important distinctions in font usage in mathematical texts. Research on developing better systems for mathematical optical character recognition crucially depends on having an extensive, high quality database of glyphs used in mathematical texts for training and test purposes. We present such a database of symbols constructed from a large set of characters available in the $\mathrm{LT}_{\mathrm{E}} \mathrm{X}$ document preparation system that can serve as a basis mathematical text recognition. We describe its integration into a prototypical system optical character recognition system for mathematics that enables the construction of LATEX source documents from mathematical documents available as images. From the lessons learned in this work we derive a road map for further research into the area of mathematical text analysis.
\end{abstract}

\section{Introduction}

There is a conspicuous need to translate the knowledge locked in the existing large body of printed scientific documents into a more accessible, searchable and versatile electronic form. A critical component in the document analysis technology required to fill this need is effective and accurate optical character recognition for type-set mathematics. However, optical character recognition (OCR) of mathematical texts poses some special problems. Unlike non-scientific text, there is a very large range of symbols commonly used. The Comprehensive Latex Symbol List [5], for example, presents 2,826 different symbols available in $\mathrm{FT}_{\mathrm{E}} \mathrm{X}$. More than that, while it is not uncommon to meet a mixture of only upright, italic and bold font faces in a non-mathematical text, the situation in mathematics is very different with a large number of font variants not only being common, but crucial to an understanding of the text because of significant semantic information being carried by often relatively subtle distinctions in font faces. Possibly because of the low current demand in the mass market for such functionality and the high cost in processing and memory overheads to provide it, commercial suppliers have not, to date, devoted a great deal of effort to 
the problems of these areas. Nevertheless, the automatic processing of scientific texts is highly desirable as their availability in electronic form would make their content more widely accessible by specialist audiences or by users with special needs.

Academic work on mathematical OCR (MOCR) and document analysis has developed since Anderson's initial work in 1968 [1], but the INFTY system [4], which is possibly the most advanced mathematical document analysis system currently extant, still relies on a mixture of a standard commercial OCR system for normal text and a custom mathematical symbol OCR system that caters for only 564 symbols (including the various alphabets) in mathematical sections. One of the problems that hinders development in MOCR is the lack of a suitable database of glyphs and symbols to use to train MOCR systems and upon which to base experimental development of scientific document analysis systems.

We have developed such a database by extracting symbols from a specially fabricated document containing approximately 5,300 different mathematical, scientific and textual symbols. This document is originally based on [5] and has been extended to cover most of the mathematical and textual alphabets and symbols currently freely available in IATEX. We have integrated the database into our own experimental MOCR system, which derives from a metric based technique for font recognition [7]. In spite of the fact that it is still only in prototype form, the system already enables us already to process mathematical documents given as images and use the database to construct $\mathrm{AT}_{\mathrm{E}} \mathrm{X}$ source files that reproduce the input documents.

Our work could be seen as approaching the same goal as [8] but from the opposite direction. [8] aims at building a ground truth set of mathematical symbols by compiling a database of characters from a selected set of mathematical articles. While our database may not enjoy the same authority as a full ground truth set, it has more breadth in that it contains most supported $\mathrm{IAT}_{\mathrm{E} X} \mathrm{Xymbols}$ rather than just the necessarily limited set contained in the publications considered. In particular, we can deal with the rapidly growing number of symbols used in diverse scientific disciplines such as computer science, logics, and chemistry.

We continually use the terms character, symbol and glyph in this document. We use the first two interchangeably to refer to the most elementary indivisible graphical element from a font directly available to a writer. A glyph, however, is more elementary in that it is a single connected component of a graphical element, one or more of which are required to make a symbol. Thus the three symbols "a", "\%" and "三" can be seen to be composed of 1, 2 and 3 glyphs, respectively.

In Sect. 2 we discuss some of the issues that must be addressed in the design of an MOCR system and their consequences for a glyph database. We describe the actual database and its contents in in Sect. 3. Section 4 discusses, using an example, the process that our experimental MOCR system applies in using the database to analyse the image of a document and reconstruct it from the database. We address some lessons learned and future work in Sect. 5 and summarise the final conclusions in Sect. 6. 


\section{Design Issues}

The aim of our work is to design a database of symbols that can be used as a basis for a wide range of research in the field of MOCR. In particular, we wish to facilitate research on MOCR without prejudicing possible approaches to the problems involved. Hence we would like our database to be as neutral as possible with respect to the pattern recognition technologies that may be employed on its contents. Furthermore, we would like to make the system such that writing code to manipulate, analyse and process the database is relatively simple so that even inexperienced users can use it for worthwhile OCR and MOCR projects. In this way, we believe, we lower the barrier to entry to research in the field.

For this reason, we have designed a simple, if rather wasteful, $\mathrm{LT}_{\mathrm{E}} \mathrm{X}$ file, where symbols are listed with identification and calibration markings. The layout of the file is designed for easy processing and our code base provides a tool to extract all the glyphs from the symbols in the file and save them as separate tiff images in a directory structure that allows easy access. An index file is also generated that, for each symbol, identifies its component glyphs and relevant information about them.

There are a number of issues that must be taken into consideration both directly for the design of the database and indirectly because of the effect they have on consequent MOCR pattern matching strategies.

Base points: The base point of a character is a distinguished position relative to the image of the symbol (but not necessarily within the bounding box of the image) that is used to align it with other symbols in a text. A document processing system aligns symbols on a line by placing them so that their base point lies on a common base line and that two neighbouring symbols are placed so the base point of the second is placed in a horizontal position relative to a point calculated as the horizontal component of the base point of the first plus the width of the first. This horizontal position can be adjusted depending on the needs for filling lines or adjusting spacing.

The base point of a symbol bears no automatic relationship to the bounding box of the symbol. For example, a symbol with a descender such as "g", "j", "p", "q" or "y", will have its base point positioned above the lower limit of its bounding box. Many symbols such as "_" or "=", have their base point positioned below the lower limit of their bounding boxes.

Thus a symbol has to be identified before its base point can be found, although the knowledge of where the baseline is together with a database of symbol baselines can be used to constrain the choice of possible matching symbols. Our database must therefore relate the base point for each symbol, and indeed, of each glyph of each symbol, to the bounding box of the symbol.

Multi-glyph symbols: Some symbols are composed of multiple glyphs. For nonmathematical OCR, this does not pose significant problems because there are relatively few such cases (mostly accents, a few punctuation symbols and an occasional symbol such as "=") and there are obvious approaches that can be 
used to deal with such exceptions. For MOCR, however, there are many more cases and, frequently, cases where different symbols share common components e.g., $\cup, \uplus, \uplus$ or $\int, \iint, \iiint, \iiint \int$ or $\prec, \precsim, \preccurlyeq, \prec, \precsim, \preceq$.

This presents a choice for a MOCR system: should it try to directly recognise symbols as a whole or should it try to recognise individual glyphs and reconstruct symbols from glyphs at a different level in the system? Since it is quick and easy to reconstruct a symbol from the component glyphs, but not to do the reverse, the database should store glyphs rather than symbols, but with the necessary information so that symbols can be conveniently reconstructed.

Duplicate glyphs: In $\mathrm{LT}_{\mathrm{E}} \mathrm{X}$, there are often multiple different fonts which have symbols which render to precisely the same graphical object at some resolution. For example there are many different instances of simple squares or circles in different fonts which are graphically indistinguishable when rendered (although they may have different base points). From the pure graphical pattern matching point of view, such objects are representatives of equivalence classes and it is the class that matters. Nonetheless, it can be significant to higher levels of mathematical document analysis as to precisely which representative it is, so the information about all such representatives must be maintained in the database with the actual final choice being made on the basis of, possibly, further contextual evidence.

Scanning resolution: Commercial, mass market OCR systems tend to be tuned to work best on relatively low resolution (200-400 dpi) images. This is possibly because of the time, memory and processing power demands that handling higher resolutions images would place on customer's resources. For normal OCR, this appears to be adequate given the relatively small symbol sets that need to be recognised and the relatively low demands for type face discrimination that current customers appear willing to put up with.

For MOCR, given the large symbol sets and the large number of fonts and typefaces that must be reliably distinguished, it seems unlikely that such resolutions will suffice. Our preliminary studies indicate that $600 \mathrm{dpi}$ may be adequate for MOCR and Suzuki has reported in [4] excellent recognition accuracy at this resolution. This is fortunate as mid to high volume scanning devices are currently readily available at this resolution but become extremely expensive at higher resolutions.

Many publishers, libraries and other organisations are currently working on digitising their collections and back catalogues. As with JSTOR [3], there seems to be a consensus on scanning at 600dpi. Therefore, for at least the foreseeable future, MOCR systems will need to be able to handle documents at this level of resolution. Nonetheless, we would like our database to be adaptable to researchers who wish to experiment with different, or even multiple resolutions.

Whatever the resolution, there will be issues of aliasing due to the limits of both the printing and the scanning technologies. 
Point size: Merely scaling a symbol from one point size to match the appropriate dimensions of the glyph at a different point size can have the effect of upsetting its weight ${ }^{1}$. For this reason, the same symbol rendered at different point sizes often have different shapes, and different approaches have been taken in OCR systems to deal with it. Some systems try to use features invariant in the type of shape changes that occur between different point sizes. Others train their systems on collections of symbols that include glyphs at a few, or even at many different point sizes. All such options should be available to users of our glyph database.

Scaling: Irrespective of the point size the symbols of the target documents were originally rendered in, the document may have been scaled via photocopying or the printing process before being scanned. This complicates the issue of correctly identifying glyphs if different point sizes are being explicitly catered for.

Unknown symbols: Whatever the extent of a glyph database, it can never be complete. Not only will obscure symbols arise from the past that have not been included, but new symbols will be designed - sometimes by an author deliberately positioning multiple symbols onto overlapping locations, sometimes by design of new fonts and symbols. A MOCR glyph database has to be easily extensible and must provide support for allowing an MOCR system to decide correctly that there is no glyph in the database that is a significant match to a target glyph in a document.

\section{A Database of Glyphs}

Our database currently consists of a set of $\mathrm{HT}_{\mathrm{E}} \mathrm{X}$ formatted documents (one per point size for $8,9,10,11,12,14,17$ and 20 points) and rendered to tiff format (multi-page, 1 bit/sample, CCITT group 4 compression) at 600dpi, and an annotation text file, automatically generated from the $\mathrm{H}_{\mathrm{E}} \mathrm{X}$ sources during formatting, containing one line for each symbol described in the $\mathrm{HT}_{\mathrm{E}} \mathrm{X}$ documents which associates the identifier of the symbol with the $\mathrm{LT}_{\mathrm{E}} \mathrm{X}$ code necessary to generate the symbol together with the information on what extra $\mathrm{I}_{\mathrm{A}} \mathrm{T}_{\mathrm{E}} \mathrm{X}$ packages or fonts, if any, are required to process the code and whether the symbol is available in math or text mode. Together with the documents, we provide Java programs to process them to extract the glyphs from the documents and store them in a suitable directory structure with one tiff file per glyph, and no more than 100 symbols per directory, and an index file containing the requisite extra information such as bounding box to base point offsets, identification of sibling glyphs in a symbol etc. We render the tiff images from the formatted $\mathrm{L}_{\mathrm{E}} \mathrm{T}$ documents using ghostview to obtain the cleanest possible images devoid of

\footnotetext{
${ }^{1}$ The weight of a symbol refers to the thickness of the strokes used to draw it. If the weights of the symbols in a font are badly matched, as, for example, you might get in a poorly designed font, the overall effect of a block of text in that font is unaesthetic and looks "unbalanced".
} 


\begin{tabular}{lll}
0953 & $\mathrm{~L}$ & \multicolumn{1}{c}{} \\
& & \\
0954 & $\mathrm{~L}$ & $\mathrm{~L}$
\end{tabular}

Fig. 1. Sample image from the database document.

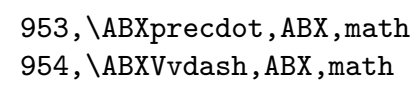

Fig. 2. Sample entries from the database annotation file.

the kind of noise, skew, distortion, scaling and machine dependent incidental problems that printing and scanning would introduce. We do not currently use a relational database to store the images and related information. However that is merely for ease of experimental development and switching to such an approach presents no difficulties.

Implementation of an MOCR program requires implementation of a glyph matching algorithm which normally requires analysing the glyphs in the database to construct a configuration set for the matcher. Use of the matcher normally requires access to the configuration set (usually quite small) but not to the actual glyphs. For our own metric based matcher [6], we generate a feature vector set from the database which can be quickly and efficiently loaded. It is intended that researchers could choose to use their own matcher and compile their own configuration set from the glyphs or, if their focus is on different levels of the mathematical document analysis process, simply use our provided matcher.

The $\mathrm{L}_{\mathrm{T}} \mathrm{T}_{\mathrm{E}} \mathrm{X}$ documents of the database enumerates all the symbols and homogenises their relative positions and sizes with the help of horizontal and vertical calibrators. The single symbols are then extracted by recognising all the glyphs a symbol consists of as well as their relative position to each other and to the calibrators. Each entry in the database thus consists of a collection of one or more glyphs together with the relative positions and the code for the actual $\mathrm{LT}_{\mathrm{E}} \mathrm{X}$ symbol they comprise. A sample of two symbol entries from a $\mathrm{HAT}_{\mathrm{E}} \mathrm{X}$ database document is shown in Fig. 1 and the corresponding excerpt from the annotation file in Fig. 2. The sans-serif "L" symbols above and to the left of the target symbols serve to identify the horizontal and vertical components respectively of the base point of the symbol. The four digit number to the left is the identification number of the target symbol which is used to relate the symbol to the appropriate line in the annotation file.

The first page of the ${ }^{\mathrm{A}} \mathrm{T}_{\mathrm{E}} \mathrm{X}$ document contains only the 10 digits and the calibration "L" symbol as registration data for a simple mini OCR matcher that 
is used to match the calibration and identification symbols while processing the rest of the document.

Among its approximately 5,300 symbols, the database contains about 1,600 mathematical symbols and 1,500 characters from different mathematical alphabets. The remaining symbols are mostly regular textual characters, accents, as well as additional scientific symbols, such as chemical or meteorological symbols.

As well as all the symbols from the standard teTeX distribution [10], we currently include the symbols from the following fonts and packages in the database: accents, amssymb, ar, bbding, bbm, bbold, bm, chemarr, chemarrow, dingbat, dsfont, esint, esvect, eurosym, euscript, fclfont, ifsym, latexsym, manfnt, mathabx, mathdots, mathrsfs, mbboard, nath, nicefrac, overrightarrow, phonetic, pifont, schwell, skak, skull, stmaryrd, suet, textcomp, tipa, trfsigns, trsym, txfonts, ulsy, undertilde, universa, upgreek, wasysym, wsuipa, yfonts, yhmath, zapfchan.

\section{Assembling $\mathrm{IAT}_{\mathrm{E}} \mathrm{X}$ Documents}

At the moment we have essentially two algorithms available to produce documents with our OCR system. The first one recognises the glyphs in the input document and matches them against the glyphs in the databases. It then takes the closest matching glyph from the database, possibly applies some scaling to it, and places it at the position in the new document that corresponds to the position of the recognised glyph in the original file. While the results of this technique are visually nearly optimal and may have applications in the area of compression of images of scientific documents, the produced file is still not very useful for further processing, such as semantic text analysis or translation of mathematical expressions into the formal input syntax of other software systems. Nonetheless, it does provide an excellent basis for testing the efficacy of our glyph matching algorithm by providing an image which we can compare with the original image via an XOR based differencing function.

The resulting document provides us with information as to where and what (equivalence classes of) glyphs have to be placed and therefore provides constraints on which symbols are involved in the document. However, since the glyphs used can also be just parts of more complex symbols and can moreover be scaled, a significant amount of extra processing is required before the actual mathematical expression that is rendered can be reconstructed.

The aim of the second algorithm is to use the $\mathrm{IAT}_{\mathrm{E}} \mathrm{X}$ commands associated by the database to the matched glyphs to assemble the final document. The algorithm roughly works in three steps:

1. The glyphs in the original document are identified.

2. an appropriate symbol is chosen from the database,

3. the $\mathrm{I}_{\mathrm{E}} \mathrm{X}$ command for that symbol is put at the correct position in the output document.

The algorithm is identical to our first one up to the point where a matching glyph is chosen. Then there are essentially two cases to consider: (a) If the glyph 
matches with a symbol that consists of that one glyph alone we can simply pick it (the result may not be the correct symbol from a semantic point of view but the formatted output should be visually indistinguishable). (b) In the case that the best match for a recognised glyph is a glyph in the database that belongs to a symbol that is composed of multiple glyphs we cannot simply take that symbol since it might introduce glyphs into the result that have no counterpart in the original document. In this case we can consider two possible conflict resolution strategies:

1. We search all closely matching glyphs for one that is the only glyph of its associated symbol.

2. We search all closely matching glyphs for one whose sibling glyphs in its symbol are also matched in the appropriate relative position.

While approach 1 might not deliver necessarily the best matching glyph, it definitely will not introduce superfluous information into the document. But in some cases it will not be possible to find a symbol that matches acceptably well with the original glyph and approach 2 might be preferable (and in general, approach 2 is, of course, more correct from a semantic perspective), which forces a search over sets of glyphs of the particular area under consideration. In our current (first) implementation we have chosen to follow approach 1 by allowing for a small error threshold when matching glyphs and giving a preference to matching single glyph symbols over multi-glyph symbols within that threshold.

Once our algorithm has decided on an appropriate symbol, it retrieves the corresponding $\mathrm{LT}_{\mathrm{EX}}$ command from the database and places it at the right position in the resulting document. Thereby it constructs a $\mathrm{LAT}_{\mathrm{E} X} \mathrm{X}$ picture environment whose measurements essentially corresponds to the bounding box given by the original document. The symbols are placed with single \put commands and, if necessary, put into math mode. In order to display symbols in the right size the algorithm uses the information provided by the database on the font size (from 5 to 20 points) of retrieved symbols. Depending on the desired point size of the final document, the command for a symbol is prefixed by the appropriate $\mathrm{H}_{\mathrm{E}} \mathrm{X}$ command for changing font sizes. For instance, in the case when the algorithm retrieves a symbol of 11 point size and we assemble a 12 point document, the prefix will be \small. In the case when it retrieves a 14 point symbol, \large will be attached. This approach somewhat limits the number of available font sizes to those made available by ${ }^{A} \mathrm{~T}_{\mathrm{E}} \mathrm{X}$ 's sizing commands. Moreover, it does not address the problem that symbols may need to be scaled horizontally and vertically differently in order to match the original glyphs. The second algorithm therefore does not give the same optimal results as the first algorithm which could insert the images with proper scaling. The right scaling for the $\mathrm{HATE}_{\mathrm{E}} \mathrm{X}$ commands will have to be addressed in future work.

We demonstrate the results of our algorithm with an example from a paper [11] we have experimented with that offers a large number of complex mathematical expressions. The particular expression we are interested in is given in Fig. 3 as it appears in the paper. The result of our OCR algorithm, as a comparison, is displayed in Fig. 5. Since the results are difficult to distinguish with the 


$$
\lambda_{u}=\sum_{v \in V} \lambda_{v} \sum_{e \in E_{u \rightarrow v}}\left(r_{\kappa(e)}\right)^{s_{1}}, \quad \sum_{u \in V} \lambda_{u}=1, \quad \lambda_{u}>0
$$

Fig. 3. Original mathematical expression image.

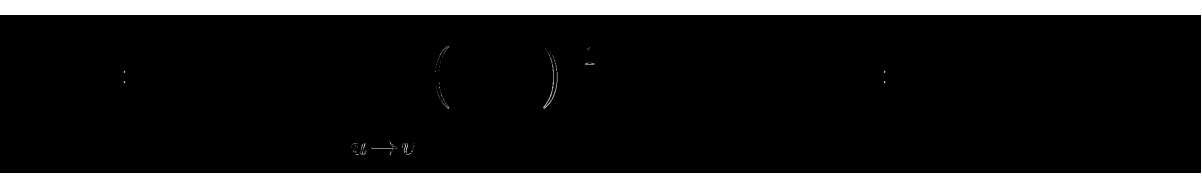

Fig. 4. Difference between Fig. 3 and Fig. 5 using XOR rendering.

$$
\lambda_{u}=\sum_{v \in V} \lambda_{v} \sum_{e \in E_{u \rightarrow v}}\left(r_{\kappa(e)}\right)^{s_{1}}, \quad \sum_{u \in V} \lambda_{u}=1, \quad \lambda_{u}>0
$$

Fig. 5. Generated mathematical expression image.

naked eye, we have combined both images using exclusive-or rendering, which is given in Fig. 4. Here, all pixels that show up in only one of the two images appear as white pixels. The difference in the rendering of the two expressions can be more easily explained when looking at the $\mathrm{LAT}_{\mathrm{E}} \mathrm{X}$ sources of the two expressions.

The source for the original expression is given in Fig. 6 whereas as the source for the output expression is displayed in Fig. 7. The latter is the input for the picture environment of dimension $3000 \times 300$ points in 12 point font size, where the unitlength is .12 points, which corresponds to $600 \mathrm{dpi}$.

If we now take a look at the symbols that are responsible for the differences in the two expressions we can see that they are caused by symbols that have not been recognised as the correct symbol or that are not properly scaled. In the latter category we have the two brackets, because large brackets in \large font size render slightly differently than in displaymath mode.

For the incorrectly recognised symbols the most obvious one is the small $v$ on the righthand side of the arrow in the subscript of the second summation sign, which is recognised as an Jupsilon. The arrow in this expression is also not recognised as an arrow in math mode but rather as one in text mode. The subscript 1 in the exponent $s_{1}$ is not recognised as a symbol from the mathrm font but as a symbol from a special text font with the \textoneoldstyle command. Furthermore, we can see that the equality sign, the only symbol here that is made up from more than one glyph, is not recognised as such but instead replaced by two vertical bar commands \HBar stacked on top of each other. Note that the $\backslash$ textoneoldstyle command belongs to the textcomp package and that the 


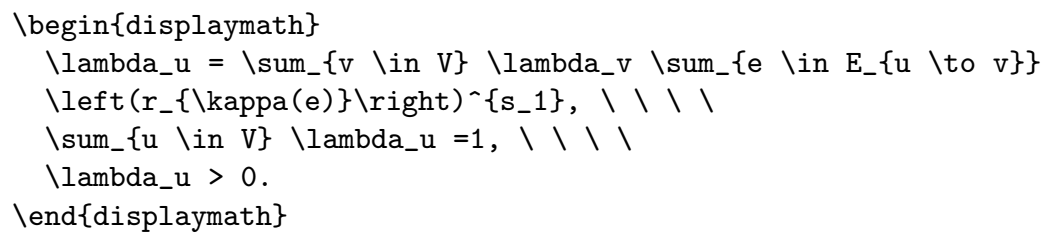

Fig. 6. $\mathrm{LT}_{\mathrm{E}} \mathrm{X}$ source for expression in Fig. 3.

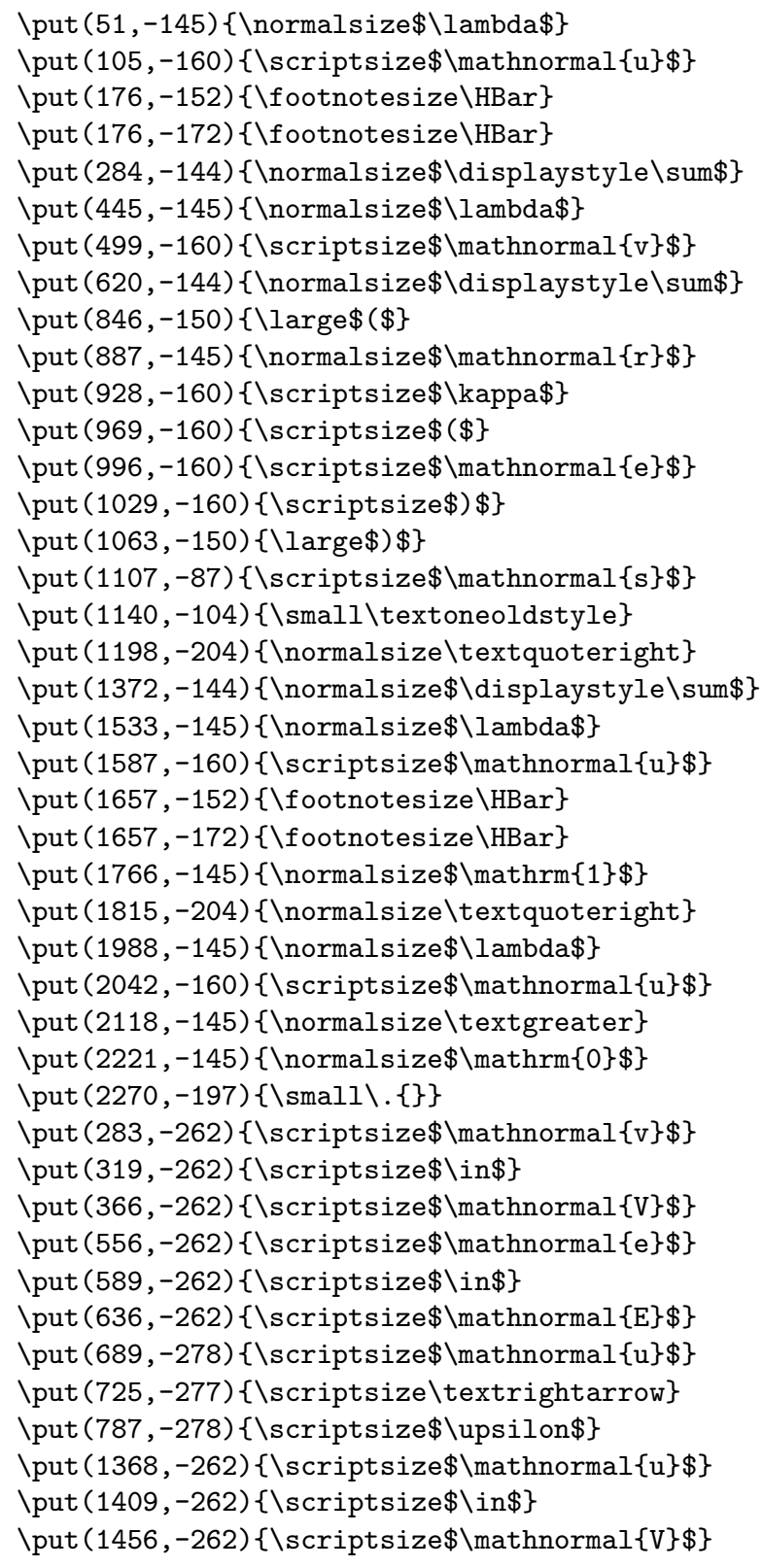

Fig. 7. $\mathrm{LT}_{\mathrm{E}} \mathrm{X}$ source for the expression in Fig. 5. 
command $\backslash$ HBar is from the package ifsym package, which is not included in the standard $\mathrm{LT}_{\mathrm{E} X}$ distribution.

\section{Discussion and Future Work}

Besides working with documents that are already compiled from actual $\mathrm{LT}_{\mathrm{E}} \mathrm{X}$ source files we are currently experimenting with scanned images of documents. In particular, we have started experimenting with articles from the Transactions of the American Mathematical Society [9]. Within the repository of the JSTOR archive [2], images of all the back issues of this journal — starting 1900 - have been made available electronically. While the results of our approach for actual LTEX documents are already nearly optimal, the reproduced files for scanned articles are still some way from a perfect translation.

Partly this is due to the fact that our implementation is new and time has not yet been available for tuning the system. In particular, we have not yet added the standard image preparation algorithms that one would expect in such a system (e.g., noise reduction filters, global and local deskewing algorithms etc.).

More seriously, our matcher is a metric based one and was originally developed on a much smaller database of glyphs. With such a small database the density of the occupied region of the metric space was low so that slightly distorted or noisy glyphs obtained from scanned images of non-LATEX documents were still closer to an excellently matching glyph in the database (if there was an appropriate match at all) than to any other glyph. With the much higher density of the metric space region we now have (because the number of entries has vastly increased but the metric distance between the furthest separated objects and the effective dimensionality of the space has not), there is a much higher probability that a disturbance in the shape of a glyph will move it into the neighbourhood of a different glyph in the database. This is an indication of the importance of having a high quality database of glyphs when designing matchers for MOCR. Now that we have such a database, we intend to investigate refinements of our feature design and matcher implementation.

It is still our central hypothesis that, because of the range and coverage of symbols available from $\mathrm{AT}_{\mathrm{E} X} \mathrm{X}$, there will be few symbols in mathematical documents, from at least the last century, that will not be matched reasonably well by a corresponding $\mathrm{AT}_{\mathrm{E} X} \mathrm{X}$ symbol. Furthermore, that a metric based symbol recogniser that works well on distorted or damaged $\mathrm{IAT}_{\mathrm{E}} \mathrm{X}$ documents, will also work well on moderately high quality images from non-LATEX mathematical documents such as can be found in the JSTOR repository [2]. This hypothesis requires proper testing.

As a first experimental implementation of an MOCR glyph database and an MOCR system, there are many missing features that need to be added and issues that need to be addressed. Some of these issues may require changes to the database, to the precompiled MOCR configuration information or just to the MOCR algorithm. As it is not yet clear which issue will require which type of change, we simply list the issues below: 
- As first experiments with papers from the American Mathematical Society suggest, we need more fonts for ordinary alphanumeric symbols. Since our database is easily extensible, adding more fonts should not be a problem.

- We currently do not have a satisfactory strategy for handling symbols of variable size which do not expand in a simple scaling based manner, e.g., square root symbols. We are currently investigating the application of affine transformation invariants in dealing with the problem.

- We do not currently have a strategy for handling touching or broken glyphs in our MOCR system. Any solution that we arrive at may require extra information obtainable from the database.

- We need to add an image pre-processing front end for noise filtering, deskewing etc.

- Currently, our MOCR system identifies what it considers to be a best match for each target glyph and reports that. This is not satisfactory for a full featured system as there may be many excellent matches at the raw glyph comparison level and it may only be higher levels of the system that can disambiguate them. For this reason we intend to replace the current behaviour with returning a lazy list, filterable on annotation information such as point size and font family, in best match order. Hence higher levels of the system could choose easily between good matches to find one more suitable to the context.

- A longer term goal is to recognise complex mathematical objects from arrangements of single symbols in order to combine them to expressions that can be stated as the sort of commands that normally appear in $\mathrm{L}_{\mathrm{E}}^{\mathrm{A}} \mathrm{\textrm {E }}$ documents. There are a number of technologies, such as graph grammar rewriters, tree transformers etc., that may be required in such tasks.

- We do not currently deal with any aspect of diagrams, even basic ones such as line or curve identification.

More generally, symbol recognition is only one part of a full document analysis system for scientific texts. There are many pairs of symbols which are visually very similar (or even identical) but which are used with different intentions. These intentions can not be disambiguated by the symbol recogniser. At best, the recogniser can return a list of symbols which, from a purely visual point of view, are credible matches for the target symbol. Ideally this list can be ordered by visual similarity to the target symbol. A naïve document analysis system might simply choose the first element in the list as the matched symbol. A more sophisticated system would choose the best match based on contextual information, visual similarity as evidenced by the ordering in the match list, and resolution of the system of constraints that arise from the possible interdependent sets of choices of surrounding symbols. The design of the interface between the recogniser and other levels of the document analysis system will be critical in obtaining a high quality and efficient result. In particular, we believe that information transfer should not be unidirectional from the recogniser to the syntax and semantic analysis subsystems. Instead there should be a flow of data in both directions so that processing on both sides of the interface can be informed by (partial) results on the other. Our future work will include research on 
such an interface with particular emphasis on avoiding compromising acceptable processing performance.

\section{Conclusion}

We have developed a large database of glyphs used in mathematical texts, which we propose as a training set for OCR systems for scientific document analysis. Together with the database, we have developed an initial, if still basic, MOCR system that demonstrates the utility of the database. We have applied it to construct $\mathrm{L}_{\mathrm{E}} \mathrm{X}$ processable source files from given images. It produced excellent results on papers formatted in $\mathrm{IAT}_{\mathrm{E}} \mathrm{X}$ and we are currently conducting similar experiments with scanned original documents.

The database is easily extensible in terms of dealing with new characters and fonts as well as multiple occurrences of effectively the same characters. We have, however, not yet fully resolved the problems arising from multi-glyph symbols.

We deal with different point sizes of characters by translating them into appropriate $\mathrm{L}_{\mathrm{E}} \mathrm{X}$ commands. While this approach does not fully solve the problem of scaling glyphs to the right size and is therefore not yet optimal, it already leads to acceptable results.

We consider the sensitivity of the recogniser to the precise shapes of the same symbol at different point sizes to be a success of the system: it is highly sensitive but, even when it chooses the wrong symbol, it chooses one which is visually very similar to the correct one. In general, it is our position that over-sensitivity can be tuned down and managed with appropriate syntactic and semantic feedback from other levels in a full document analysis system. However, we believe that under-sensitivity is much more difficult to compensate for.

Although the current database and associated software is still under active development, and in particular the MOCR system is still only a prototype, we already have promising results in our experiments and expect later versions of the system to be a valuable tool for the transcription of traditional mathematical documents into electronically managed knowledge.

Availability The experimental version of the database is available upon request from the authors.

\section{References}

1. Robert H. Anderson. Syntax-Directed Recognition of Hand-Printed Twodimensional Mathematics. PhD thesis, Harvard University, Cambridge, MA, January 1968. Shorter version in M. Klerer and J. Reinfelds (eds), Interactive Systems for Experimental Applied Mathematics, pages 436-459, 1968, Academics Press.

2. The JSTOR scholarly journal archive. http://www.jstor.org/.

3. The JSTOR production process. http://www.jstor.org/about/process.html. 
4. M.Suzuki, F.Tamari, R.Fukuda, S.Uchida, and T.Kanahori. Infty - an integrated ocr system for mathematical documents. In C.Vanoirbeek, C.Roisin, and E. Munson, editors, Proceedings of ACM Symposium on Document Engineering, pages 95-104, Grenoble, France, 2003.

5. Scott Parkin. The comprehensive latex symbol list. Technical report, CTAN, 29 September 2003. available at twww.ctan.org.

6. Alan Sexton and Volker Sorge. Database-driven mathematical character recognition. In Josep Llados and Liu Wenyin, editors, Graphics Recognition, Algorithms and Applications, LNCS, Hong Kong, August25-26 2005. Springer Verlag. to appear.

7. Alan P. Sexton, Alison Todman, and Kevin Woodward. Font recognition using shape-based quad-tree and kd-tree decomposition. In 3rd International Conference on Computer Vision, Pattern Recognition and Image Processing, pages 212-215, Atlantic City, USA, Feb. 2000. Appears in Vol 2 of the collected proceedings of JCIS 2000, the Fifth Joint Conference on Information Sciences.

8. Masakazu Suzuki, Seiichi Uchida, and Akihiro Nomura. A ground-truthed mathematical character and symbol image database. Technical report, Faculty of Mathematics, Kyushu University, 6-10-1 Hakozaki, Higashi-ku, Fukuoka-shi, 812-8581 Japan, 2004. Available at http://www.inftyproject.org/AboutInftyCDB-1.pdf.

9. Transactions of the American Mathematical Society. Available as part of JSTOR at http://uk.jstor.org/journals/00029947.html.

10. The teTeX homepage. http://www.tug.org/teTeX/.

11. Peter Tiño and Barbara Hammer. Architectural Bias in Recurrent Neural Networks: Fractal Analysis. Neural Computation, 15(8):1931-1957, 2003. available at http://www.cs.bham.ac.uk/ pxt/PAPERS/rnn.frac.nc.fin.ps.gz. 